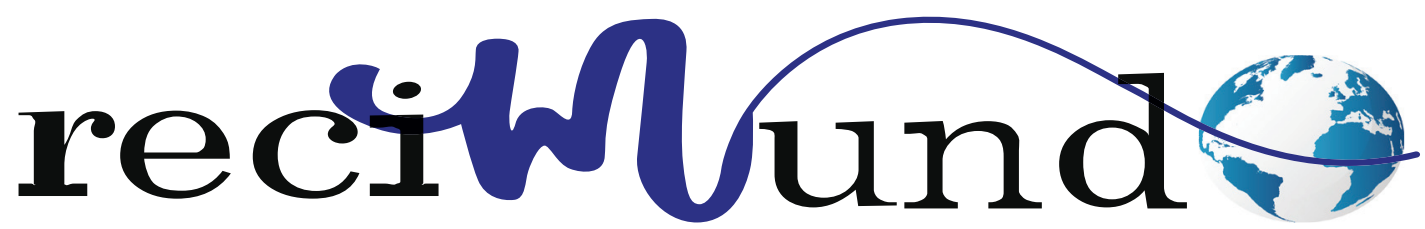

Revista Científica Mundo de la Investigación y el Conocimiento

DOI: 10.26820/recimundo/5.(4).oct.2021.53-64

URL: https://recimundo.com/index.php/es/article/view/1297

EDITORIAL: Saberes del Conocimiento

REVISTA: RECIMUNDO

ISSN: 2588-073X

TIPO DE INVESTIGACIÓN: Artículo de revisión

CÓDIGO UNESCO: 32 Ciencias Médicas

PAGINAS: 53-64

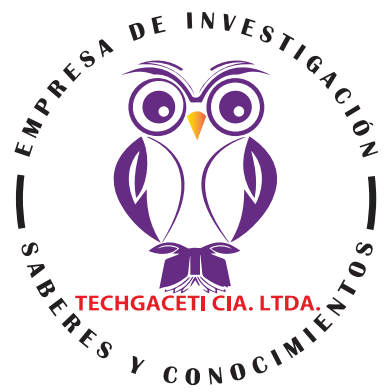

\title{
Astrocitoma, diagnóstico y tratamiento
}

Astrocytoma, diagnosis and treatment

Astrocitoma, diagnóstico e tratamento

René David Astudillo Mancero1; Jhonny Edgar Narváez Álvarez; Paola Alejandra Villagrán Herrero3;

Alejandra Patricia Lafuente González ${ }^{4}$

\section{RECIBIDO: 02/09/2021 ACEPTADO: 20/09/2021 PUBLICADO: 30/10/2021}

1. Médico Cirujano; Especialista en Medicina Interna; Investigador Independiente; Quito, Ecuador; dr.reneastudillo.medinterna@gmail.com; (D) https://orcid.org/0000-0002-2534-5684

2. Médico General en Funciones Hospitalarias; Quito, Ecuador; jenarvaezsdq@gmail.com; (DD https://orcid.org/0000-00015554-7319

3. Médico General en Funciones Hospitalarias; Quito, Ecuador; dra.villagranh@gmail.com; (iD https://orcid.org/0000-0002$5282-6248$

4. Médico General en Funciones Hospitalarias; Quito, Ecuador; apatylg@hotmail.es; iD https://orcid.org/0000-0002-76993059

\section{CORRESPONDENCIA}

René David Astudillo Mancero

dr.reneastudillo.medinterna@gmail.com

Quito, Ecuador

() RECIMUNDO; Editorial Saberes del Conocimiento, 2021 


\section{RESUMEN}

Los astrocitomas son tipos de tumores cerebrales, que se producen por un crecimiento anómalo y descontrolado de tejido glial, presentes en el sistema nervioso y que alimentan y dan soporte a las neuronas. La metodología de la investigación, es una revisión bibliográfica apoyada en medios electrónicos como páginas web (SCOPUS, PubMed, Biblioteca Cochrane, Google Scholar), en donde se encontró una amplia variedad de artículos de investigación que abordan la temática estudiada, el objetivo es recopilar la información más relevante de dichos autores para realizar una síntesis bien completa, que pueda ayudar a futuras investigaciones. Los astrocitomas son una patología bastante compleja, los cuales están clasificados en diferentes grados y tipos. En la mayoría de los casos el diagnostico está basado en pruebas de imágenes, específicamente de resonancias magnéticas, que tienen un alto grado de exactitud, también se utilizan en otro casos tomografías computarizadas. Los tratamientos son de índole quirúrgicos, combinados con radioterapias, quimioterapias y el uso de fármacos. Hay casos donde la resección del tumor no puede ser completa, lo que puede conllevar a otra intervención quirúrgica, de estar la porción que quede del tumor en un sector que se pueda acceder quirúrgicamente, de no ser así, como ya se ha mencionado se utiliza radioterapia o quimioterapia, según sea el caso. Luego de realizados los procedimientos quirúrgicos, los pacientes deben continuar con sus valoraciones, cada cierto tiempo, para evitar el riesgo de la reaparición del tumor.

Palabras clave: Tumor, Neurona, Astrocitoma, Glial, Resección.

\section{ABSTRACT}

Astrocytomas are types of brain tumors, which are produced by an abnormal and uncontrolled growth of glial tissue, present in the nervous system and which feed and support neurons. The research methodology is a bibliographic review supported by electronic media such as web pages (SCOPUS, PubMed, Cochrane Library, Google Scholar), where a wide variety of research articles were found that address the subject studied, the objective is to collect the most relevant information from these authors to make a complete synthesis, which may help future research. Astrocytomas are a fairly complex pathology, which are classified into different grades and types. In most cases the diagnosis is based on imaging tests, specifically magnetic resonance imaging, which have a high degree of accuracy, computed tomography scans are also used in other cases. The treatments are surgical in nature, combined with radiotherapies, chemotherapies and the use of drugs. There are cases where the resection of the tumor cannot be complete, which may lead to another surgical intervention, if the remaining portion of the tumor is in a sector that can be accessed surgically, if not, as already mentioned, it is used radiotherapy or chemotherapy, as the case may be. After performing the surgical procedures, patients should continue with their evaluations, from time to time, to avoid the risk of the tumor reappearing.

Keywords: Tumor, Neuron, Astrocytoma, Glial, Resection.

\section{RESUMO}

Astrocitomas são tipos de tumores cerebrais, produzidos por um crescimento anormal e descontrolado do tecido glial, presente no sistema nervoso e que alimenta e sustenta os neurônios. A metodologia da pesquisa é uma revisão bibliográfica apoiada em meios eletrônicos como páginas web (SCOPUS, PubMed, Cochrane Library, Google Scholar), onde foram encontrados diversos artigos de pesquisa que abordam o assunto estudado, o objetivo é coletar os mais relevantes informações desses autores para uma síntese completa, o que pode auxiliar pesquisas futuras. Os astrocitomas são uma patologia bastante complexa, que são classificados em diferentes graus e tipos. Na maioria dos casos, o diagnóstico é feito com base em exames de imagem, especificamente a ressonância magnética, que apresentam alto grau de acurácia, a tomografia computadorizada também é utilizada em outros casos. Os tratamentos são de natureza cirúrgica, combinados com radioterapia, quimioterapia e uso de medicamentos. Há casos em que a ressecção do tumor não pode ser completa, o que pode levar a outra intervenção cirúrgica, se o restante do tumor estiver em um setor que possa ser acessado cirurgicamente, caso contrário, como já mencionado, utiliza-se radioterapia ou quimioterapia, conforme o caso. Após a realização dos procedimentos cirúrgicos, os pacientes devem prosseguir com suas avaliações, periodicamente, para evitar o risco de reaparecimento do tumor.

Palavras-chave: Tumor, neurônio, astrocitoma da glia, ressecção. 


\section{Introducción}

En el campo de la medicina y especialmente en el área de oncología, al hacer referencia a los astrocitoma, se identifican como toda aquella neoplasia o tumor formada principalmente por astrocitos, uno de los principales tipos de células gliales que alimentan y dan soporte a las neuronas. Dicho tumor es una masa de astrocitos que se produce ante un crecimiento y proliferación anómala, patológica y descontrolada de uno de los tipos de tejido glial presentes en el sistema nervioso, tratándose de uno de los principales tipos de tumor cerebral (Cruz, Vega, \& González, 2019, pág. 856). Se clasifican de acuerdo a la World Health Organization (WHO) en lesiones de bajo grado (I y II) y lesiones de alto grado (III y IV), siendo las de bajo grado más frecuentes que las de alto grado en niños (Popó \& Maya, 2017, pág. 107).

Los tumores grado I tienen bajo potencial proliferativo y tienen posibilidad de cura al ser resecados quirúrgicamente. Los tumores grado II son tumores infiltrantes, pero de baja actividad proliferativa celular, tienden a recurrir y en algunos casos, como los gliomas, a progresar a grados superiores (III y IV). Los tumores grado III son lesiones con evidencia histológica de malignidad y los grado IV tienen evidencia de malignidad citológica con predisposición a necrosis y están relacionados con una evolución rápida y fatal de la enfermedad, como lo es el glioblastoma. Estos tumores grado III y IV son denominados de "alto grado" o "malignos" (Eric Jesús, Francisca, \& Ivanis Idael, 2021).

\section{Tipos y características de los astrocitomas}

Tabla 1. Clasificación de los astrocitomas

\begin{tabular}{cc}
\hline Localizados (15\%) & Difusamente infiltrados (85\%) \\
\hline Astrocitoma pilocítico & Astrocitoma difuso de bajo grado \\
Astrocitoma pilomixoide & Glioma pontino intrínseco difuso \\
Astrocitoma subependimal de células gigantes & Astrocitoma anaplásico \\
Xantoastracitoma pleomorfico & Glioblastoma multiforme \\
& Gliosarcoma \\
& Gliomatosi cerebri \\
\hline
\end{tabular}

Fuente: (Domenech, y otros, 2018)

- En el astrocitoma fibrilar cuya celularidad es baja a moderada, el núcleo celular se encuentra oval e irregular e hipercromatico, la mitosis es ausente, citoplasma perinuclear escaso y los bordes celulares son apenas reconocibles, mientras que su tipificación es dada por presentar abundante proteína ácido glial fibrilar.

- El astrocitoma protoplásmico, presenta el núcleo celular redondo y rico en cromatina y en ocasiones se observa mitosis al igual que degeneración microcitica y el bajo contenido de proteína ácido glial fibrilar ratifica su tipificación.

- El astrocitoma gemistocítico, está compuesto de células poligonales angulares con abundante citoplasma eosinofilico, los procesos celulares se observan cortos y anchos, los núcleos celulares se observan redondos a ova- 
les excéntricamente localizados, la mitosis es rara y la infiltración linfocítica es más común que en otras variantes de astrocitomas, su tipificación está conformada por presentar un elevado nivel de proteína ácido glial fibrilar.

- El astrocitoma de medio grado o anaplásico está caracterizado por las características de anaplasia así como incremento de la celularidad, pleomorfismo, atipia nuclear y actividad mitótica, esta variante de astrocitoma puede ser confundido con el de alto grado de malignidad sin embargo la proliferación vascular y la necrosis no se presentan en el anaplásico.

- El astrocitoma de alto grado o también denominado glioblastoma es la variante que, en adición a las características del anaplásico presenta proliferación vascular y necrosis, estas neoplasias causan considerables daños cerebrales como la herniación fatal, presenta elevada celularidad, las células tumorales son pobremente diferenciadas y polimórficas, ocasionalmente se observan células agigantadas multinucleadas al igual que la proliferación vascular glomeruloide y necrosis prominente, macroscópicamente son circunscritos que varían de coloración y consistencia debido a la degeneración, necrosis y hemorragia (Velázquez-Ordoñez, y otros, 2015, págs. 2-3).

Dependiendo de la ubicación y del tipo de tumor medular pueden aparecer diferentes síntomas, especialmente debido a que el tumor crece y afecta la médula espinal, los nervios que la rodean o los vasos sanguíneos. Los astrocitomas presentan una incidencia de 1 por cada 100.000 habitantes en nuestro medio. Estos tumores causan gran nivel de discapacidad y sufrimiento al paciente por su sintomatología y daño medular (Rojas Carmenathy, Castellanos Bertot, \& Massó Rodríguez, 2019, pág. 642). Su localización más común es supratentorial, la ubicación a ambos la- dos del tentorio (supra e infra) es todavía más inusual, de hecho, no hay una frecuencia consensuada de esta forma de presentación. En general se diferencian en lesiones multicéntricas y multifocales (Chávez-López, García-Cisneros, Zarate-Méndez, \& García, 2019, pág. 146). 


\section{Diagnóstico}

Tabla 1. Diagnósticos de algunos tipos de Astrocitomas

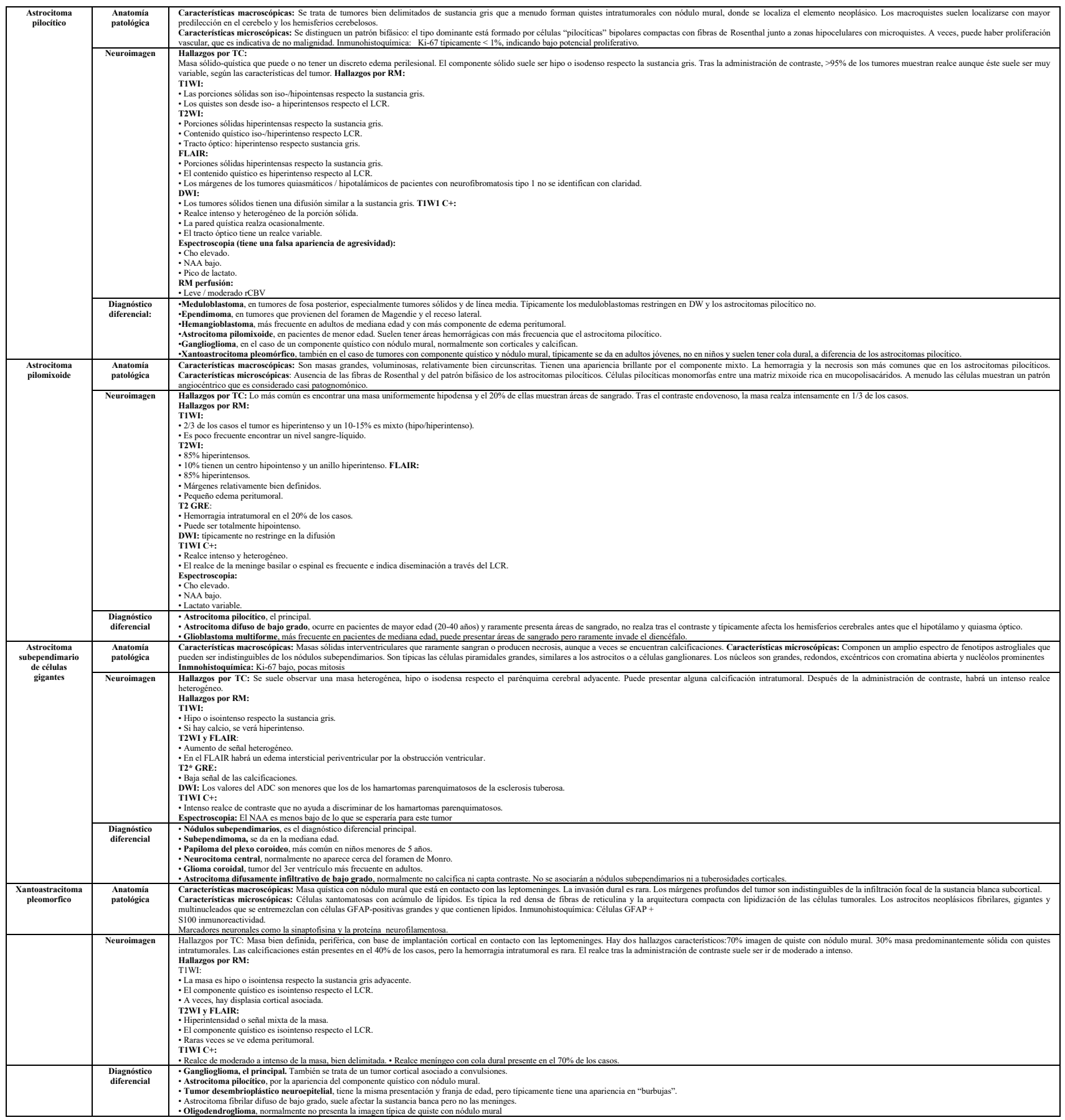

Fuente: Elaboración Propia. Tomado de (Domenech, y otros, 2018). 


\section{Síntomas}

En cuanto a los síntomas que presenta el astrocitoma están en función de varios factores, pero especialmente de la localización del tumor. Si están en el cerebro suelen causar convulsiones, dolores de cabeza y vómitos. Los que están en la médula espinal pueden causar cierta discapacidad en la zona del cuerpo afectada por el tumor. Por ello, el médico ha de realizar como primer paso un examen neurológico en el que habla con el paciente y obtenga información acerca de su comportamiento y estado general (Cruz, Vega, \& González, 2019, pág. 866).

\section{Algunos tratamientos de astrocitoma, de- pendiendo de su grado o tipo}

El tratamiento principal de los glioblastomas y de los astrocitomas anaplásico ha sido, y continúa siendo, la mayor resección quirúrgica posible del tumor, documentada mediante resonancia magnética nuclear (RMN) dentro de las $72 \mathrm{~h}$ de la cirugía. El tratamiento adyuvante con radioterapia asociado a quimioterapia, basada en compuestos alquilantes como la carmustina (BCNU) o la temozolomida (TMZ) han demostrado que pueden incrementar la supervivencia. El tratamiento con inhibidores de angiogénesis como el anticuerpo monoclonal dirigido contra el receptor del factor de crecimiento epidérmico (EGFR) fue aprobado en 2009 para el tratamiento de GB recurrentes tras encontrarse altas tasas de respuesta de supervivencia libre de progresión. La inmunoterapia pasiva con anticuerpos monoclonales (AcM) es de las más efectivas contra el EGFR. Actualmente existen varios AcM contra ese receptor registrados para el tratamiento de algunos tumores sólidos epiteliales: Cetuximab (AcM quimérico), nimotuzumab (AcM humanizado) y Panitumumab (AcM humano) (Elzaurdín Mora \& Lara Fernández, 2019, pág. 3).
Según los últimos resultados del estudio de Stupp. Incluso con tratamiento combinado agresivo la mediana de supervivencia es sólo de 12-15 meses, con una supervivencia media a los cinco años inferior al 5\% Generalmente, la evolución tras el tratamiento primario de los gliomas de bajo grado es a la recidiva en gliomas de alto grado (evolución a glioma secundario), y los gliomas de alto grado tienden a recidivar tras obtener respuesta al tratamiento inicial. Los tumores del SNC de alto grado, debido a su carácter infiltrante después de cirugías radicales y alas dosis de radioquimioterapia, desarrollan la recidiva tumoral en el 60-90\% de los casos, bien en el punto de origen o a 2-3 cm alrededor del lugar inicial, siendo los factores pronósticos más importantes la edad menor de 55 años, el estado general del paciente, medido por la escala de Karnosfky (80- 100) o Zubrod ECOG menor o igual a dos, tamaño tumoral menor o igual a $4 \mathrm{~cm}$, localización frontal y resección total macroscópica.

Sin embargo el pronóstico todavía es desfavorable y en algunos casos solamente se puede iniciar tratamiento sintomático paliativo en las Unidades de Cuidados Paliativos. Los tratamientos combinados de radioquimioterapia concomitante en recidivas, como los esquemas de dosis utilizados por Schonekaes y Mucke dosis (20-30 Gy/1,2 Gy/2 fracciones al día) y quimioterapia con dosis de temozolamida de 400 mg/durante 5 días/cada 28 días alcanzan una mediana de duración de respuesta de 7 meses (Muñoz Carmona, Faga Cantamessa, García-Salazar, Gómez Millán, \& Bayo, 2005, pág. 44)

\section{Alternativas de tratamiento para astro- citomas subependimarios de células gi- gantes \\ Inhibidores de la vía mTOR}

Hay distintos fármacos inhibidores de mTOR: rapamicina (sirolimus), RAD001 (everolimus) y CCl-779 (temsirolimus). El 
primero en ser identificado fue sirolimus, un antibiótico macrólido producido por la fermentación de Streptomyces hygroscopicus inicialmente utilizado como antifúngico, posteriormente se identificaron sus propiedades antiproliferativas e inmunodepresoras. Everolimus es una sustancia de administración oral, estructuralmente semejante a sirolimus y con propiedades similares pero con mayor estabilidad y solubilidad, y con una farmacocinetica más favorable. Tanto sirolimus como everolimus son tratamientos potenciales para complejo de esclerosis tuberculosa, ya que inhiben la via mTOR. Otros importantes mecanismos de acción son el efecto antitumoral y la capacidad de reducir la expresión de los procesos angiogenicos, a través del factor de crecimiento endotelial que promueve la neovascularización e incrementa el crecimiento y el desarrollo de tumores sólidos. La sobrerregulación de este factor es responsable de la gran vascularización de los tumores en el complejo de esclerosis tuberosa (Ruiz, y otros, 2015).

\section{Astrocitoma fibrilar difuso}

En este mismo orden de ideas, se puede decir que las opciones terapéuticas en los astrocitoma fibrilar difuso son: observación, cirugía, radioterapia y quimioterapia. La observación estricta, con controles radiológicos periódicos, se recomienda en pacientes asintomáticos o mínimamente sintomáticos, e incluso en aquellos con crisis epilépticas al diagnóstico, pero bien controladas con tratamiento médico. La observación está contraindicada en aquellos pacientes cuya sintomatología no se controla con tratamiento médico. Por ello, la cirugía permite la obtención de histología para llegar al diagnóstico definitivo y caracterización molecular del tumor, y es la primera opción terapéutica en la mayoría de los casos. Sin embargo, aun en el caso de conseguirse una resección macroscópicamente completa, debe mantenerse un estrecho control, dado que, en pacientes <40 años intervenidos de as- trocitomas de bajo grado no pilocíticos, se ha evidenciado una tasa de progresión tumoral de más del 50\% a los 5 años. El momento óptimo para iniciar la radioterapia y las dosis más adecuadas para el tratamiento aún son objeto de discusión. En general, la radioterapia puede diferirse en pacientes en buena situación clínica, siempre que se haga un seguimiento cuidadoso. Los pacientes mayores de 40 años, los pacientes con tumores grandes irresecables y los que presentan déficit neurológico, son habitualmente tratados con radioterapia precoz. En cuanto al uso de quimioterapia, aunque no se dispone de evidencia firme para su uso adyuvante postquirúrgico (se ha observado beneficio en la supervivencia libre de progresión, pero no en la supervivencia global), hay ensayos clínicos prospectivos en marcha para evaluar esta indicación (Cruz, Vega, \& González, 2019, pág. 869).

\section{Metodología}

La metodología de la investigación, es una revisión bibliográfica apoyada en medios electrónicos como páginas web (SCOPUS, PubMed, Biblioteca Cochrane, Google Scholar), en donde se encontró una amplia variedad de artículos de investigación que abordan la temática estudiada, el objetivo es recopilar la información más relevante de dichos autores para realizar una síntesis bien completa, que pueda ayudar a futuras investigaciones.

\section{Resultados}

\section{Caso clínico}

Popó \& Maya (2017), en su trabajo de invetigacion, presentan caso clinico de paciente femenina de 14 años de edad, que acude a centro medico con referencias de cuadro clinico de 2 años de evolucion consistente en cefalea tipo pulsátil progresiva en el tiempo, hasta ser de intensidad 9/10 en la escala análoga del dolor localizada en la región frontotemporal izquierda, la cual se

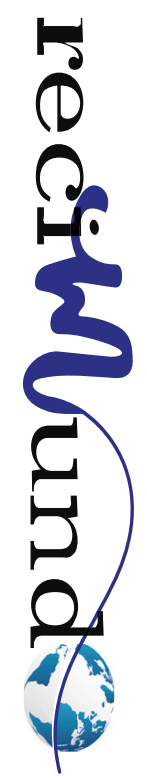


irradiaba a región retro-ocular ipsilateral, en ocasiones la despertaba en la noche y le impedía conciliar el sueño. A su vez presentaba los siguientes síntomas asociados: náuseas, vomito, tinitus, disestesias ocasionales en miembros superiores y disminución de la agudeza visual.

\section{Exámenes realizados}

- Tomografía Axial Computarizada (TAC) de cerebro simple que mostró imagen de masa intraventricular sobre el tálamo, principalmente en el atrio ventricular derecho con efecto compresivo local, no había hidrocefalia asociada ni compromiso del septum pelucidum, la lesión presentaba densidad no homogénea, el parénquima encefálico era de aspecto normal, sin evidencia colecciones extra-axiales compresivas.

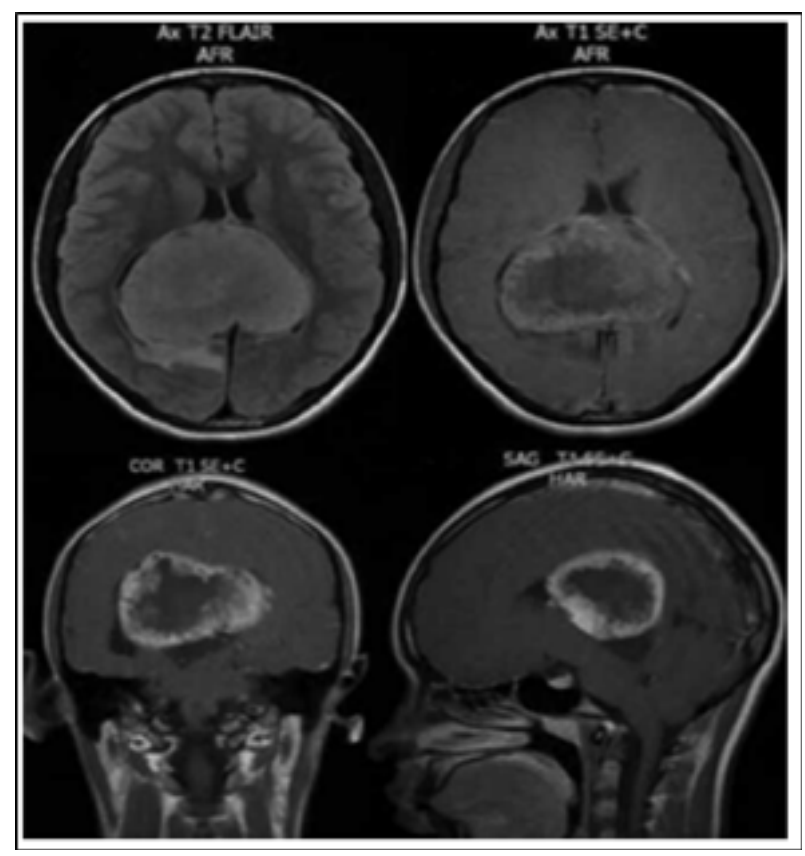

Figura 1. RM Cerebral: tumor intraventricular en el atrio que compromete ambos ventrículos laterales, el cual realza con el contraste de manera heterogénea con hipodensidad en su parte central hace compresión sobre el diencéfalo y la región pineal, dimensiones aproximadas de 50x78×56 mm; ejerce también importante efecto compresivo sobre el sistema ventri- cular en particular los atrios, con desplazamiento hacia anterior del mesencéfalo y puente, asociado a edema vasogénico parietal derecho

Fuente: (Popó \& Maya, 2017).

\section{Diagnóstico}

- Por hallazgos en TAC cerebral simple se consideró neoplasia de posible origen glial y se iniciaron medidas anti-edema, de neuroprotección y se solicitó una RM cerebral.

\section{Tratamiento}

- Se decidió llevar a la paciente a cirugía, se realizó craneotomía parieto-occipital derecha, abordaje transcortical al atrio ventricular con evidencia de extensa lesión del atrio ventricular bilateral de $6 \times 8 \times 7 \mathrm{~cm}$ vascularizada, color gris y friable. Se realizó resección amplia subtotal por relación de la lesión con venas cerebrales internas, se dejó catéter ventricular para monitoreo de PIC y vigilar sangrado, anticonvulsivantes y esteroides. Se realizó TAC cerebral simple de control a las 24 horas post-operatorio

\section{Evolución}

- La paciente presentó adecuada evolución postoperatoria, sin deterioro neurológico, no cambios en la memoria ni alteración visual o sensitiva; cedió la hipertensión intracraneal y mejoraron los síntomas por los cuales consulto. La ventriculostomía se retiró al segundo día del postoperatorio, y por la buena evolución clínica se decidió continuar manejo ambulatorio con analgésicos.

- Se realizó control por consulta externa a las 2 semanas de postoperatorio; la paciente refirió sentirse bien, sin presencia de síntomas neurológicos, sin déficit focal. Se indicó nuevo control a los 5, 9 y 
15 meses del postoperatorio con estudio de RM cerebral con contraste, las cuales muestran lesión residual en relación con la parte posterior del atrio ventricular hacia el esplenio del cuerpo calloso y el fórnix por encima de la región pineal; sin presencia de progresión de la lesión residual. Se propuso a la madre y la paciente realizar un segundo tiempo quirúrgico para completar la resección o dar manejo expectante observacional; se decidió en conjunto continuar con observación dadas las características del tumor y el buen estado clínico y neurológico de la paciente (Popó \& Maya, 2017, págs. 108-110).

\section{Caso II}

Rojas Carmenathy, Castellanos Bertot, \& Massó Rodríguez (2019), presentan caso de paciente femenina de 20 años de edad, acudió al servicio de Neurocirugía porque hace más menos un año presentó un dolor en la región lumbar que comenzó de forma leve y que luego fue aumentando hasta hacerse insoportable, el mismo se irradiaba a la porción anterolateral del muslo derecho hasta el dorso del pie. Sólo se aliviaba con la administración de diclofenaco sódico. A su vez presento: constipación, retención urinaria, dolor lumbar y calambres en las piernas.

\section{Exámenes realizados}

- Resonancia magnética que mostró una imagen hiperdensa a nivel de D12-L5.

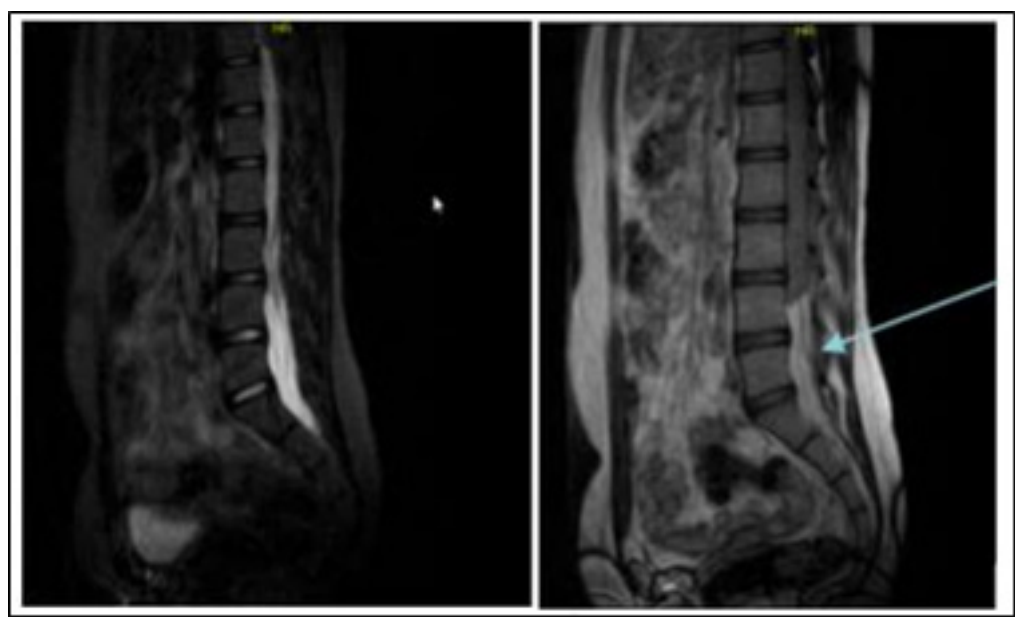

Figura 2. Resonancia magnética nuclear. Imagen hiperdensa D12-L5

Fuente: (Rojas Carmenathy, Castellanos Bertot, \& Massó Rodríguez, 2019).

\section{Diagnóstico}

- Astrocitoma grado II-III.

\section{Tratamiento}

- Dado el diagnóstico se decidió tratar de forma quirúrgica a la paciente. Se realizó laminectomía de D12-L5, durotomía y extracción tumoral. Se realizó fijación transpedicular con tornillos y barras de titanio ubicados en T12-L1-L3-L4. Cierre por planos y drenaje perivertebral. Todo esto en diez horas y media de operación bajo neuromonitorización estricta.

\section{Evolución}

- La paciente presentó una rehabilitación favorable pues al segundo día de opera-

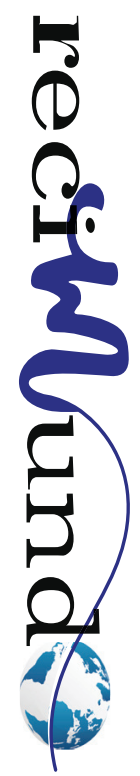


da la misma se podía poner en bipedestación cosa que con técnicas convencionales no lo hubiera hecho. Recibió varias sesiones de quimioterapia y tuvo completa remisión de su sintomatología (Rojas Carmenathy, Castellanos Bertot, \& Massó Rodríguez, 2019, págs. 642644).

\section{Caso III}

- Popó \& Maya (2017), en su trabajo de inestigacion, presenta caso de paciente masculino de 6 años de edad, que viene padeciendo desde hace 3 años disminución de la agudeza visual progresiva, cefalea intensa ocasional, fiebre intermitente, vómitos, deformidad en extremidades inferiores y proptosis derecha.

\section{Exámenes realizados}

- Se realizó biomicroscopía en la que se encontraron los siguientes datos: Segmento anterior sin alteraciones, no se encontraron Nódulos de Lisch. El fon- do de ojo bajo midriasis farmacológica en ambos ojos reportó: retina aplicada, adecuado brillo macular y papila óptica con excavación de 0.3×0.3, importante palidez de nervios ópticos (características compatibles con atrofia óptica secundaria a compresión), emergencia central de los vasos, no datos de edema papilar ni hemorragias asociadas.

- Tomografía Axial Computada de Órbita y Cráneo la cual reportó tumoración intraorbitaria derecha, superior, que desplazaba inferiormente el globo ocular y afectaba tejido óseo circundante. Se complementó el estudio con una RM de cráneo en la que además de la tumoración, se reportó la presencia de encefalocele orbitario, por lo que se decidió realizar abordaje quirúrgico en conjunto con el servicio de neurocirugía.

\section{Diagnóstico}

- Astrocitoma Pilocítico de Bajo Grado (Grado I OMS).

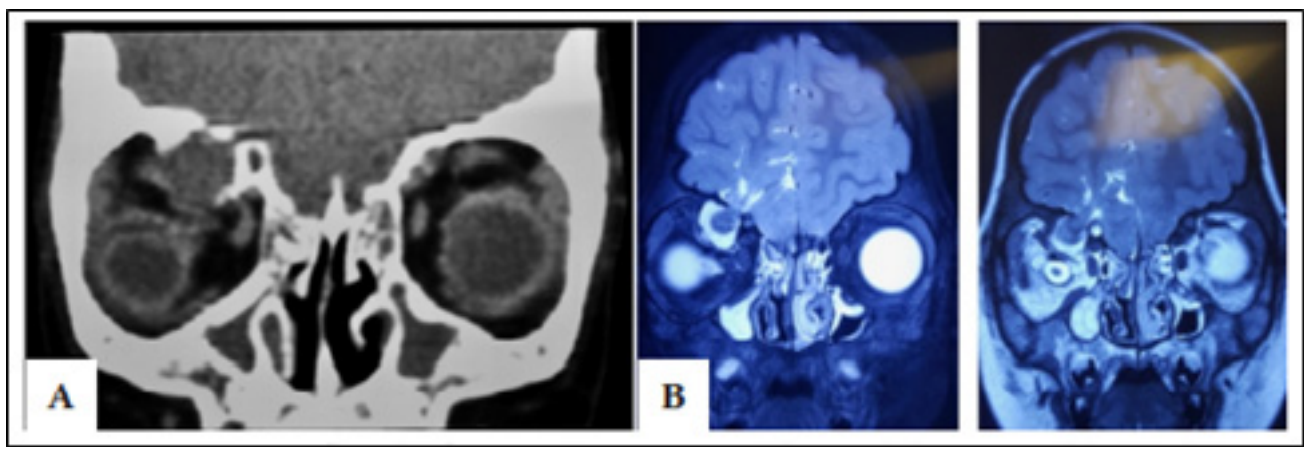

Figura 3. A: Tomografía Axial Computada, corte coronal. Se aprecia importante lisis del tejido óseo perilesional, además de desplazamiento interno del globo ocular. B: Resonancia magnética, corte coronal. Se observa herniación de tejido encefálico a órbita derecha.

Fuente: (Lemus-Cruz, González-Pérez, Sánchez-Orozco, Iñiguez-Soto, \& Castellanos-Franco, 2017). 


\section{Tratamiento}

- Se realizó procedimiento de acceso transcraneal, en donde se observó una lesión ocupativa en órbita derecha de aspecto homogéneo, la cual se resecó.

\section{Evolución}

- Se valoró en el postquirúrgico con una evolución satisfactoria, sin ser necesaria terapia coadyuvante, presentó disminución de la proptosis y la exotropia y mejoría en la movilidad ocular, sin embargo no mostró mejoría en la agudeza visual por la atrofia de nervio óptico (Lemus-Cruz, González-Pérez, Sánchez-Orozco, Iñiguez-Soto, \& Castellanos-Franco, 2017, pág. 83).

\section{Conclusiones}

Los astrocitomas son una patología bastante compleja, los cuales están clasificados en diferentes grados y tipos. En la mayoría de los casos el diagnostico está basado en pruebas de imágenes, específicamente de resonancias magnéticas, que tienen un alto grado de exactitud, también se utilizan en otro casos tomografías computarizadas. Los tratamientos son de índole quirúrgicos, combinados con radioterapias, quimioterapias y el uso de fármacos.

Hay casos donde la resección del tumor no puede ser completa, lo que puede conllevar a otra intervención quirúrgica, de estar la porción que quede del tumor en un sector que se pueda acceder quirúrgicamente, de no ser así, como ya se ha mencionado se utiliza radioterapia o quimioterapia, según sea el caso. Luego de realizados los procedimientos quirúrgicos, los pacientes deben continuar con sus valoraciones, cada cierto tiempo, para evitar el riesgo de la reaparición del tumor. Hay que dejar en claro que, el no abordaje rápido de un astrocitoma puede ocasionar la evolución del mismo a un grado, que pueda ocasionar la muerte del paciente (grados III y IV).

No hay que olvidar que al existir diferentes tipos de astrocitomas, la valoración del paciente, debe ser individualizada, para tomar la decisión que mejor convenga al paciente, y así poder realizar el tratamiento adecuado que logre la recuperación total del mismo.

\section{Bibliografía}

Chávez-López, J. A., García-Cisneros, R., Zarate-Méndez, A., \& García, S. (2019). Astrocitoma anaplásico multifocal sincrónico con localizacion lobar atípica. Revista de Especialidades Médico-Quirúrgicas, 23(3), 145.

Cruz, G. C., Vega, E., \& González, R. (2019). Astrocitoma fibrilar difuso. RECIMUNDO, 3(3), 853-875.

Domenech, M. R., Ortega, M., Mas, A., Jordi, J., Pont, G., \& Sala, C. (2018). Correlación radio-patológica en el diagnóstico de astrocitomas localizados. Seram.

Elzaurdín Mora, R. A., \& Lara Fernández, N. (2019). Uso de nimotuzumab en paciente con astrocitoma anaplásico. Revista Cubana de Medicina, 58(4).

Eric Jesús, A. V., Francisca, D., \& Ivanis Idael, C. (2021). ASTROCITOMA FIBRILAR DIFUSO GRADO II EN EDAD PEDIÁTRICA. A PROPÓSITO DE UN CASO. In cibamanz2021.

Lemus-Cruz, C. V., González-Pérez, G., Sánchez-Orozco, A., Iñiguez-Soto, M., \& Castellanos-Franco, T. (2017). Astrocitoma Pilocítico orbitario. Revista Médica MD, 8(1), 81-84.

Muñoz Carmona, D. M., Faga Cantamessa, C., García-Salazar, M., Gómez Millán, J., \& Bayo, E. (2005). Nuevas perspectivas en el tratamiento paliativo del glioblastoma multiforme y astrocitoma anaplásico recidivado con implantes de carmustina. Oncología (Barcelona), 28(5), 43-51.

Popó, H. R., \& Maya, J. (2017). Astrocitoma Pilocítico Ventricular: una presentación poco frecuente. Neurociencias Journal, 24(2), 106-114.

Rojas Carmenathy, S., Castellanos Bertot, Y., \& Massó Rodríguez, A. (2019). Astrocitoma medular de alto grado no infiltrante. Revista Información Científica, 98(5), 640-647.

Ruiz, M., Sánchez, S., Medina, A., Rea, A., Sanromán, R., García, J., \& del Mar Sáez, M. (2015). Astrocitoma subependimario de células gigantes asociado a complejo de esclerosis tuberosa: reco-

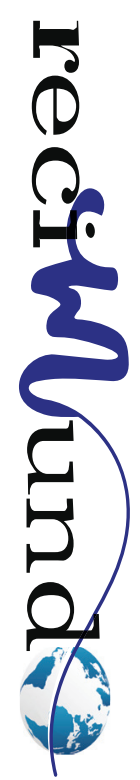


mendaciones para el diagnóstico oportuno y tratamiento. Acta Pediátrica de México, 36(1), S1-S25.
Velázquez-Ordoñez, V. A., Valladares-Carranza, B., González-Herrera, L., Barbosa-Mireles, M., Fajardo-Muñoz, R., Zamora-Espinosa, J., \& Talavera-Rojas, M. (2015). Estudio de caso de sindrome neurologico asociado a astrocitoma. REDVET. Revista Electrónica de Veterinaria, 16(5), 1-8.

\section{CITAR ESTE ARTICULO:}

Astudillo Mancero, R. D., Narváez Álvarez, J. E., Villagrán Herrero, P. A., \& Lafuente González, A. P. (2021). Astrocitoma, diagnóstico y tratamiento. RECIMUNDO, 5(4), 53-64. https://doi.org/10.26820/recimundo/5.(4).oct.2021.5364 